NASA Technical Memorandum 105892
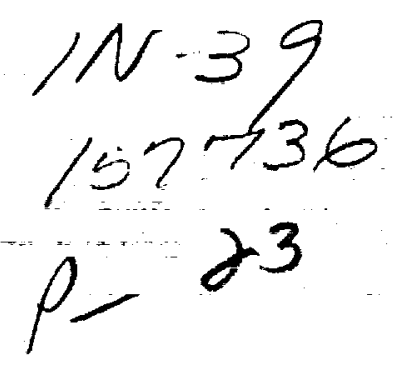

\title{
Mapping Methods for Computationally Efficient and Accurate Structural Reliability
}

Michael C. Shiao

Sverdrup Technology, Inc.

Lewis Research Center Group

Brook Park, Ohio

and

Christos C. Chamis

National Aeronautics and Space Administration

Lewis Resēarch Center.

Cleveland, Ohio

Prepared for the

33rd Structures, Structural Dynamics and Materials Conference

Dallas, Texas, April 13-15, 1992

\footnotetext{
(NASA-TM-105892) MAPPING METHODS FOR COMPUTATIONALLY EFFICIENT AND ACCURATE STRUCTURAL RELIABILITY (NASA) $23 \mathrm{p}$
} 
$\ldots+\ldots$

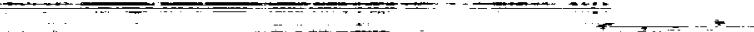

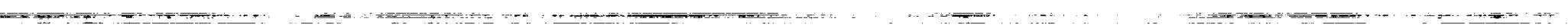




\title{
MAPPING METHODS FOR COMPUTATIONALLY EFFICIENT AND ACCURATE STRUCTURAL RELIABILITY.
}

\author{
by \\ Nichael C. Shiao \\ NASA Lewis Research Center Group \\ Sverdrup Technology, Inc. \\ Cleveland, Ohio \\ and \\ Christos C. Chamis \\ NASA Lewis Research Center \\ Cleveland, Ohio
}

\begin{abstract}
Mapping methods are developed to improve the accuracy and efficiency of probabilistic structural analyses with coarse finite element meshes. The mapping methods consist of : (1) deterministic structural analyses with fine (convergent) finite element meshes, (2) probabilistic structural analyses with coarse finite element meshes, (3) the relationship between the probabilistic structural responses from the coarse and fine finite element meshes, and (4) a probabilistic mapping. The results show that the scatter in the probabilistic structural responses and structural reliability can be efficiently predicted using a coarse finite element model and proper mapping methods with good accuracy. Therefore, large structures can be efficiently analyzed probabilistically using finite element methods.
\end{abstract}




\section{Introduction}

It has been recognized in the structures community that uncertainties in the structural parameters as well as in the service environment need to be considered in the evaluation of structural integrity/reliability. Probabilistic structural analyses are formal methods to include those uncertainties. However, these methods are inherently computationally intensive due to the large number of deterministic analyses required to accurately simulate the effect of the uncertainties on the desired structural response (stress, displacement, frequency, etc.) necessary for structural reliability assessment. Moreover, modern structures are often analyzed by finite element methods. Probabilistic structural analyses using finite element models can be economically performed if relatively coarse meshes are used. Finite element analyses using coarse meshes not only raise questions regarding the convergence on the deterministic structural responses (using mean structural parameters and mean loads) but also significantly alter the true probability distributions of the structural responses. It is important, therefore, to evaluate the influence of mesh coarseness on the accuracy of the probabilistic structural responses and the structural reliability. Recently, several alternatives to orercome the problems were examined at NASA Lewis Research Center. A methodology is developed to perform a probabilistic mapping on the results from a probabilistic structural analysis with a coarse finite element mesh. In this paper, these alternatives are presented. The theoretical background and the computational procedure are also described. Four numerical examples were analyzed to demonstrate the effectiveness and accuracy of this methodology. 
Theoretical Background

The accuracy of the probabilistic structural responses is most affected by two factors. The first one is the statistical accuracy of the probability distributions of the random variables involved in the analysis. The second one is the coarseness of the finite element models. The random variables are independent of the coarseness of the finite element model and are assumed to be accurately described. Therefore, the error of the probabilistic structural response for a given finite element mesh is directly caused by the coarseness of the model. In order to derive the mapping methods, a coarse finite element mesh must be chosen in such a way that this model reflects proper structural behavior with a tolerable error, for example, 10 to $20 \%$ of error. Since the structural responses from coarse and fine finite element meshes are simulated through the same structural mechanics and are influenced by the same random variables, high correlation between them is expected and a fully correlated condition is assumed in the following derivation. Therefore, the linear relationship between the structural responses from fine and coarse finite element meshes is represented by Eq.(1).

$$
X_{f}=A X_{c}+B
$$

where $X_{f}$ and $X_{c}$ are the probabilistic structural responses from fine and coarse meshes respectively. The constants $A$ and $B$ are determined by using the probabilistic potential energy variational principle developed by Liu, Mani and Belytschko ' and different orders of correction. The potential energy variation principle states 
that the mean and the standard deviation of the probabilistic structural response for a given finite element mesh are

$$
E(X)=X^{\circ}-\sum_{k=1}^{N}\left(X_{k}^{\prime \prime} \sigma_{k}^{2}\right)
$$

and

$$
\sigma_{X}=\sqrt{\sum_{k=1}^{N}\left(X_{k}^{\prime}\right)^{2} \sigma_{k}^{2}}
$$

where $\sigma_{k}$ is the standard deviation of the $k^{\text {th }}$ independent random variable; $N$ is the number of independent random variables; $X$ is the probabilistic structural response for a given finite element mesh; $X^{o}$ represents the deterministic structural response which is computed using mean values of the associated primitive random variables; $X_{k}^{\prime}$ and $X_{k}^{\prime \prime}$ are defined in equations (4) and (5).

$$
\begin{gathered}
X_{k}{ }^{\prime}=\partial X / \partial l_{k} \\
X_{k}{ }^{\prime \prime}=\partial^{2} X / \hat{c} l_{k}^{-2}
\end{gathered}
$$

where $U_{k}^{\prime}$ is $k^{\text {th }}$ independent random variable.

In the following, three relationships between structural responses from coarse and fine finite element models are derived based on different assumptions. 
(1) Shift Mapping

Shift mapping is a first moment correction method. Let

$$
Y_{f}=X_{f}-E\left(X_{f}\right)
$$

and

$$
Y_{c}=X_{c}-E\left(X_{c}\right)
$$

where $X_{f}$ and $X_{c}$ denote the probabilistic structural responses simulated using fine and coarse finite element meshes respectively. First order correction can be achieved by letting

$$
Y_{f}=Y_{c}
$$

Substituting Eqs.(2) and (3) into Eq.(8), we obtain

$$
\begin{aligned}
X_{f}=X_{c}-X_{c}^{o}+X_{f}^{o} & +\sum_{k=1}^{N}\left(X_{c}\right)_{k}{ }^{\prime 2} \sigma_{k}^{2} \\
& -\sum_{k=1}^{N}\left(X_{f}\right)_{k}{ }^{\prime \prime} \sigma_{k}^{2}
\end{aligned}
$$


The last two terms in Eq.(9) are related to the second derivatives of structural responses to each independent random variable. If linear relationships between the structural responses and the independent random variables exist, the second derivatives become zero. Therefore, Eq.(9) can be simplified as

$$
X_{f}=X_{c}-X_{c}^{o}+X_{f}^{o}
$$

This means that the probability distributions of structural responses simulated using a fine finite element mesh can be obtained by a simple shift on the abscissa of the probability distributions of structural responses simulated using a coarse finite element mesh. The shift amount is the difference between the deterministic values from coarse and fine finite element meshes. However, if a linear relationship does not exist, Eq.(10) is only an approximation. This approximation will be reasonable only if the coarse model causes a small error, say less than $10 \%$, or the relation between structural response and random variables is approximately linear. From this analysis, the constants $A$ and $B$ in Eq.(1) are shown in Eq.(11).

$$
\begin{aligned}
& A=1 \\
& B=-X_{c}^{o}+X_{f}^{o}
\end{aligned}
$$

(2) Ratio Mapping

Ratio mapping is a second moment correction method. Letting 


$$
Z_{f}=\frac{X_{f}-E\left(X_{f}\right)}{\sigma_{X_{f}}}
$$

and

$$
Z_{c}=\frac{X_{c}-E\left(X_{c}\right)}{\sigma_{X_{c}}}
$$

and setting the normalized structural responses $Z_{c}$ and $Z_{f}$ from coarse and fine finite element meshes to be equal, we obtain

$$
\frac{X_{f}-E\left(X_{f}\right)}{\sigma_{X_{f}}}=\frac{X_{c}-E\left(X_{c}\right)}{\sigma_{X_{c}}}
$$

Substituting Eqs.(2) and (3) into Eq.(14), the relationship between the structural responses from coarse and fine finite element meshes can be developed as follows. Let

$$
r_{1}=\frac{X_{f}^{o}}{X_{c}^{o}}
$$

and

$$
r_{2}=\sqrt{\frac{\sum\left(X_{f}\right)_{k}^{\prime 2} \sigma_{k}^{2}}{\sum\left(X_{c}\right)_{k}^{\prime 2} \sigma_{k}^{2}}}
$$


Substitute eqs.(15) and (16) into eq.(14), we obtain

$$
\begin{array}{r}
X_{f}=r_{2} X_{c}-r_{2}\left(X_{c}^{o}-\Sigma\left(X_{c}\right)_{k}{ }^{\prime \prime} \sigma_{k}^{2}\right) \\
+\left(X_{f}^{o}-\Sigma\left(X_{f}\right)_{k}{ }^{\prime \prime} \sigma_{k}^{2}\right)
\end{array}
$$

For a given coarse model which reflects proper structural behavior, the ratio $r_{2}$ is dominated by several key or sensitive random variables. This ratio can be considered as a weighted average of the individual ratio of the first or second derivative of the structural responses from fine and coarse models. Let the structural responses from fine and coarse models be represented by Eqs.(18) and (19).

$$
X_{f}=X_{f}^{o}+\sum_{i=1}^{N} a_{i} U_{i}+\sum_{j=1}^{N} b_{j} l_{j}^{2}
$$

and

$$
X_{c}=X_{c}^{o}+\sum_{i=1}^{N} c_{i} U_{i}+\sum_{j=1}^{N} d_{j} U_{j}^{2}
$$

where $a_{i}, b_{1}, c_{i}$ and $d$, are constants determined numerically. We assume 


$$
\frac{X_{f}^{o}}{X_{c}^{o}} \simeq \frac{a_{i}}{c_{i}} \simeq \frac{b_{j}}{d_{j}} \quad i, j=1, N
$$

Since, fine and coarse structural models are subjected to the same loads and same random variables with similar structural behavior. Also, in the limit, the ratios in equation (20) is equal to 1 when the coarse model approach to the fine model. Substituting Eq.(20) into Eq.(16), it is found that

$$
r_{1} \simeq r_{2} \simeq \frac{X_{f}^{o}}{X_{c}^{o}}
$$

Equation (21) will be verified later by the numerical examples. Substituting Eq.(21) into Eq.(17), we obtain

$$
X_{f} \simeq \frac{X_{f}^{o}}{X_{c}^{o}} X_{c}
$$

Therefore, the constant $A$ and $B$ in Eq.(1) are

$$
\begin{aligned}
& A=\frac{X_{f}^{o}}{X_{c}^{v}} \\
& B=0
\end{aligned}
$$


The ratio mapping method represents the fact that not only the mean values are different but also the scatter around the mean can be either wider or narrower. Therefore, the normalized structural responses from both models are considered to be equal in this method.

\section{(3) Mixed Mapping}

For a very coarse finite element mesh on which neither shift mapping nor ratio mapping works, a mixed mapping method is proposed. Mixed mapping can be considered as an average value of the structural responses predicted by shift and ratio mapping methods. The relationship between the structural responses from fine and coarse finite element meshes are represented by Eq.(24)

$$
X_{f} \simeq \frac{1}{2}\left[1+\frac{X_{f}^{o}}{X_{c}^{o}}\right] X_{c}+\frac{X_{f}^{o}-X_{c}^{o}}{2}
$$

Computational Procedure

(1) Probability Distribution

The probability distributions of the converged structural responses are simulated by the following steps.

Siep 1 : compute the convergent deterministic structural response using fine mesh r.odel. 
Step 2 : simulate the probability distribution of the structural response using a coarse finite element mesh.

Step 3 : determine the probabilistic relationship between the coarse and fine finite element responses.

Step 4 : compute the convergent probability distribution of the structural response with the results obtained from steps (1) to (3) by a probability mapping.

(2) Probability of Damage Initiation

Crack initiation occurs when the stress is greater than the strength of the material. The probability distribution of the strength is simulated using the multifactor interaction equations as described by Boyce and Chamis ${ }^{2}$. The probabilistic stress of a given finite element mesh is simulated by Shiao and Chamis ${ }^{3}$. The convergent stress is then simulated according to the computational procedure described previously. The probability of damage initiation is computed by the following equation.

$$
\begin{aligned}
P_{f} & =P(\text { stress }>\text { strength }) \\
& =\int_{-\infty}^{+\infty} F_{s h}(x) f_{s s}(x) d x
\end{aligned}
$$


where $F_{s n}$ is the cumulative distribution function of the strength; $f_{s s}$ is the probability density function of the stress.

\section{Numerical Examples and Discussion}

Four examples are studied to verify this methodology. The computer code NESSUS (Numerical Evaluation of Stochastic Structures Under Stress) ${ }^{4}$ is used to perform the probabilistic structural analyses. Maximum effective stress and the first buckling load from coarse and fine meshes using different mapping methods are simulated in the following examples.

In the first example, a cantilever plate subjected to lateral pressure is analyzed as shown in Fig.(1). Plate thickness and the uniform pressure are considered to be random variables. Although the coarse finite clement mesh underestimates the stress by $25 \%$, the prediction of the cumulative distribution function of the stress using ratio mapping with a coarse finite element mesh compares very well with the result from the fine finite element mesh as shown in Fig.(2). In this example, the ratio $r_{1}$ and $r_{2}$ in Eqs.(15) and (16) are equal as derived in Eq.(21). Their values are

$$
r_{1}=r_{2}=1.326
$$

It is found that the shift mapping method provides good mean values but not the scatter. The probability of damage initiation is shown in Fig.(3). This study indicates the accuracy predicted in the tail region of the probability distributions of the 
structural responses. Again, the prediction from ratio mapping gives an excellent result.

In the second example, a buckling analysis of a simply supported composite plate is performed. The plate geometry and the loading conditions are shown in Fig.(4). The random variables considered are the coefficients of the stiffness matrix for the force resultant-generalized strains relations from laminate theory. The probabilistic distributions of those coefficients are computed by the NASA Lewis in-house computer code PICAN (Probabilistic Integrated Composite Analyzer). In this example, the buckling load is overestimated by $11 \%$. However, the buckling load is predicted well by ratio mapping as shown in Fig.(5). $r_{1}$ and $r_{2}$ are found to be equal again. Their values are

$$
r_{1}=r_{2}=0.89
$$

In the third example, a cantilever plate subjected to thermal/mechanical loads as shown in Fig.(6) is analyzed. Three random fields (uncertainties) are considered. They are thickness, modulus and temperature. Each field consists of correlated nodal random variables. The loads at the free end are also considered to be random. The structural parameters, such as modulus and strength, deteriorate under aggressive service environments. In this example, even with a poor mesh $16 \%$ error in the deterministic response), the probabilistic distribution of the structural response using a coarse finite element mesh adjusted by the ratio mapping method compare very well with those using fine inite element mesh as shown in Fig.(7). The probability 
of damage initiation is also predicted well by the same method as shown in Fig.(8). In addition, the ratio of the computational time using coarse and fine meshes is about $1: 20$ as shown in Table (1). Therefore, tremendous computational time is saved with this methodology.

In the final example, a tapered cantilever plate with variable thickness is studied. The geometry and the loading conditions are shown in Fig.(9). The uncertainties considered are the same as those in the previous example. The cumulative distribution function of the effective stress in Fig.(10) and the probability of damage initiation in Fig.(11) are predicted very well by ratio mapping.

\section{Concluding Remarks}

In conclusion, the mapping methods are developed to perform probabilistic structural analyses with coarse finite element meshes. It is found that (1) the shift mapping works well only with a very good coarse mesh (i.e. error is less than $5 \%$ ); (2) ratio mapping accurately predicts the probability distribution and the structural reliability even using a very coarse mesh; and, (3) results from mixed mapping always lie between those from shift and ratio mapping. Using this methodology, good accuracy can be achieved and computational time will be minimized. Therefore, the dilemma experienced using either coarse or fine meshes for the probabilistic structural analyses, especially for large structures, is resolved. 


\section{References}

1. Liu, W. K, Mani, A., Belytschko, T., "Finite Element Methods in Probabilistic Mechanics", Probabilistic Engineering Mechanics, 1987, Vol. 2, No. 4, pp. $201-213$

2. L., Boyce, C.C. Chamis, "Probabilistic Constitutive Relationships for Cyclic Material Strength Models", 29 th SDM Conference, Williamsburg, Virginia, 1988

3. Shiao, M. C., Chamis, C. C., "A Methodology for Evaluating the Reliability and Risk of Structures under Complex Service Environments", Proceedings of the 31st Structures, Structural Dynamics and Materials Conference, 1990, pp. 1070-1080."

4. "Probabilistic Structural Analysis Methods for Select Propulsion System Structural Components (PSAM)", Vols. 1-3, NASA CR-185125-Vol-1,-2,-3, 1989 


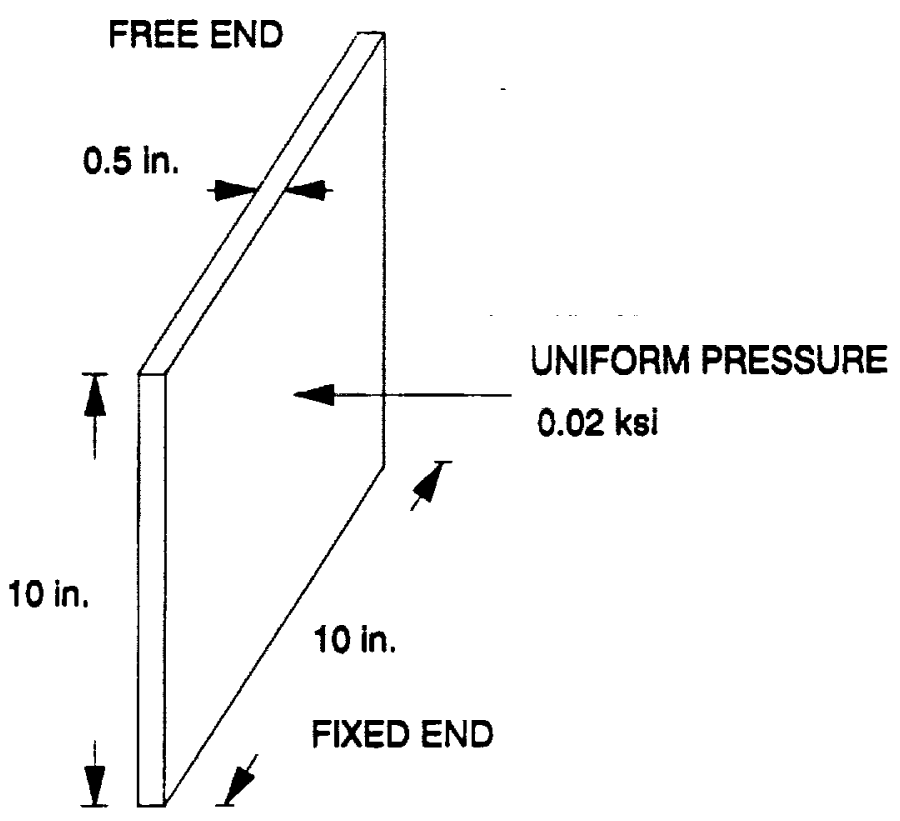

Fig. 1. Geometry and Loading Condition of a Square Cantilever Plate (Example 1)

CUMULATIVE
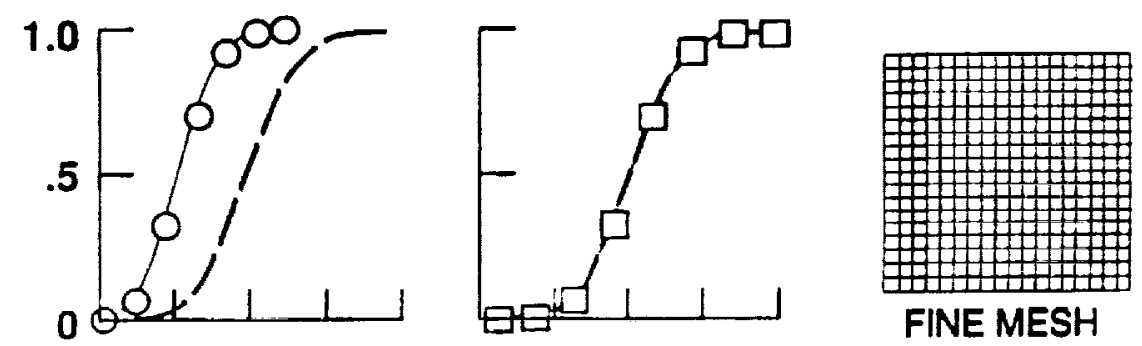

DISTRIBUTION
FUNCTION
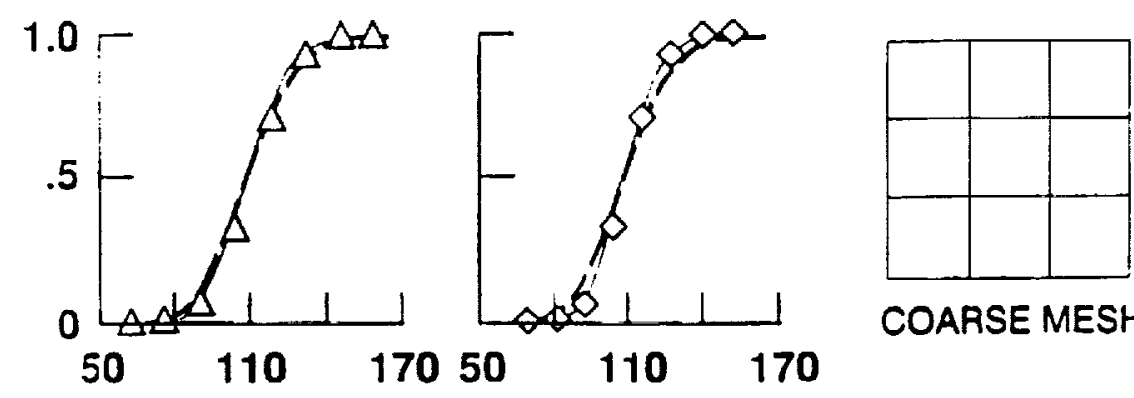

COARSE MESH

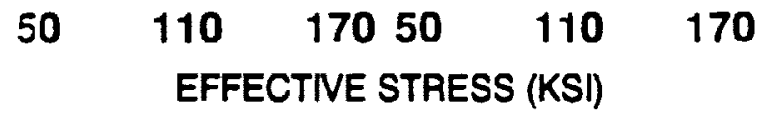

- - FINE MESH

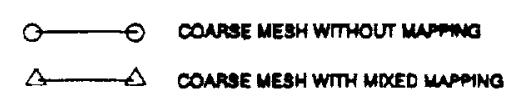

$\square$

COARSE MESH WTTH RUTO MUPPING COARSE MESH WITH SHIFT MUPPINO

Fig. 2. Comparison Between the Cumulative Distribution Functions of the Effective Stress from Different Mapping Methods (Example 1) 


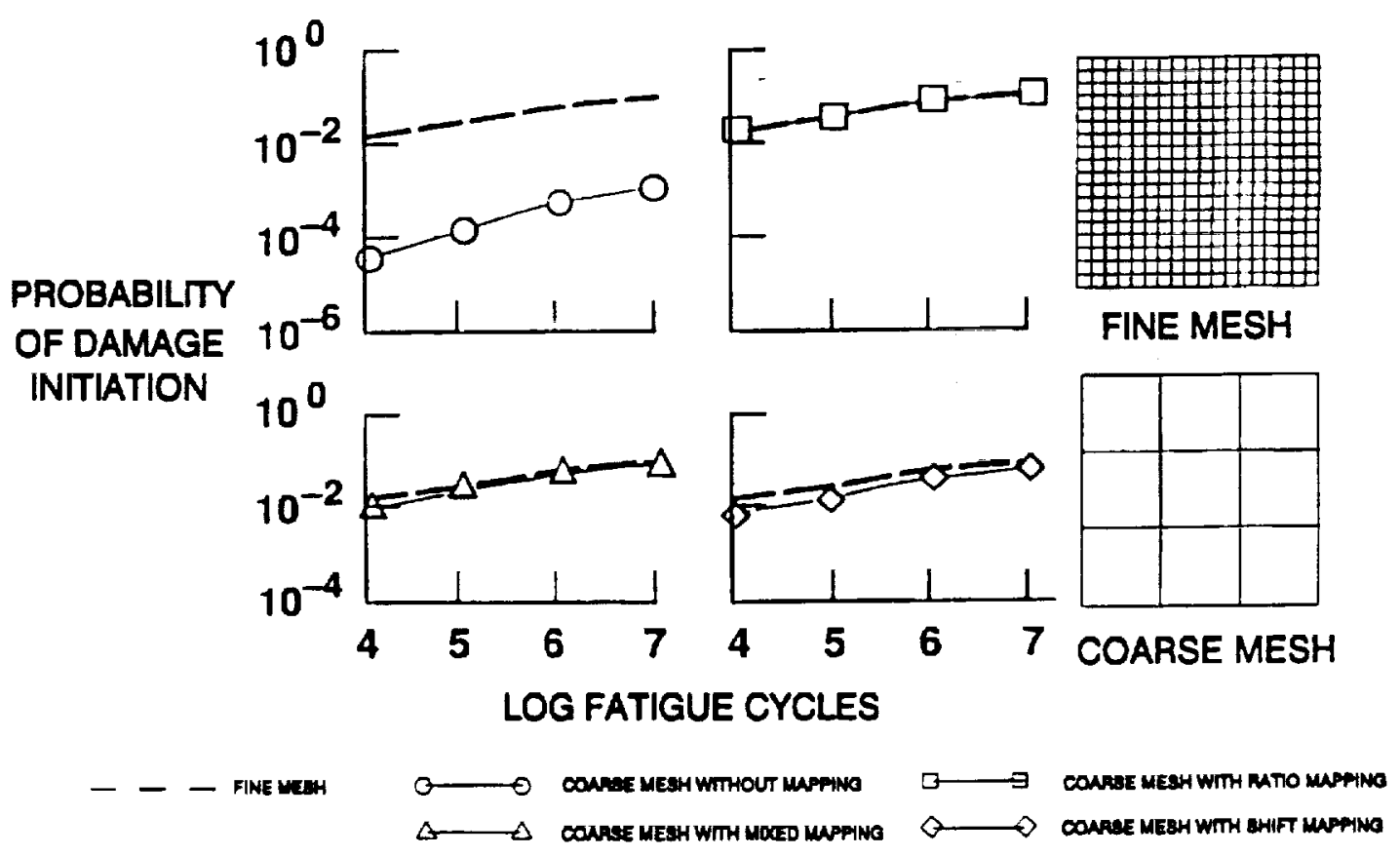

Fig. 3. Comparison Between the Probability of Damage Initiation from Different Mapping Methods (Example 1)

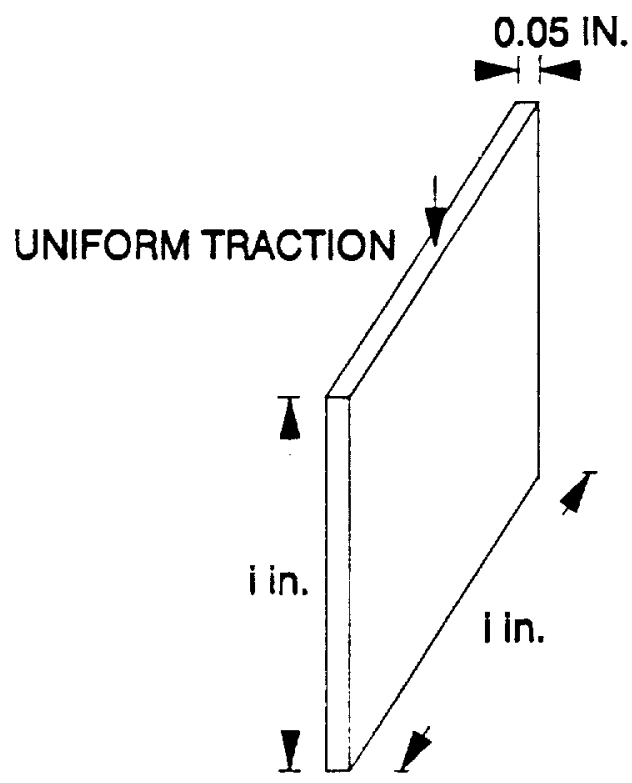

Fig. 4. The Geometry and Loading Condition of a Simply Supported Composite Plate (Example 2) 
CUMULATIVE DISTRIBUTION
FUNCTION
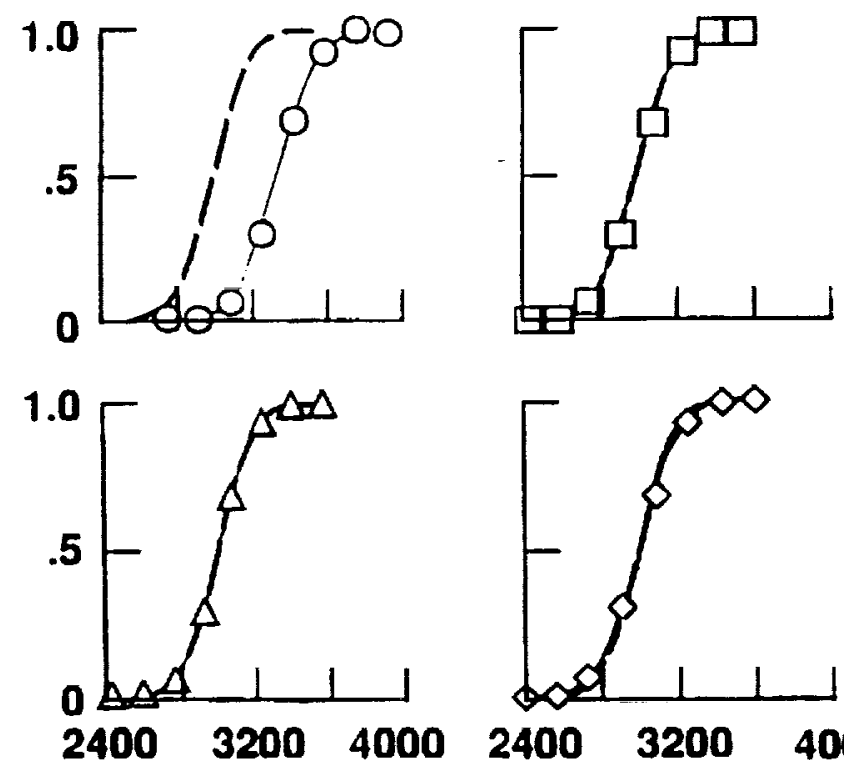

BUCKLING LOAD (Ib/in.)

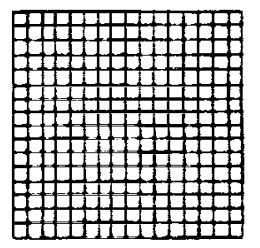

FINE MESH

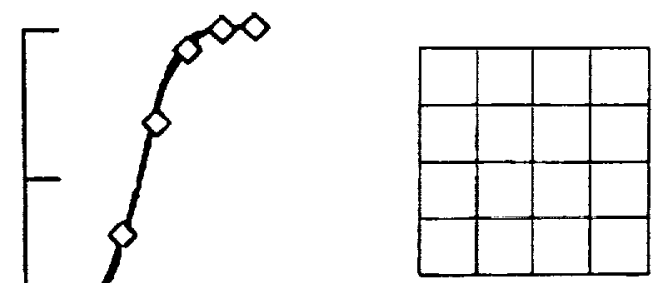

COARSE MESH

- - FINE MESH

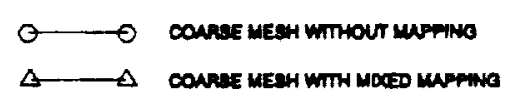

$\sigma-\theta$
$\theta-\theta$

COARSE MESH WTH RATIO MAPANO COARE MESH WTH SHIFT MUPPHNO

Fig. 5. Comparison Between the Cumulative Distribution Functions of the First Buckling Load (Example 2)
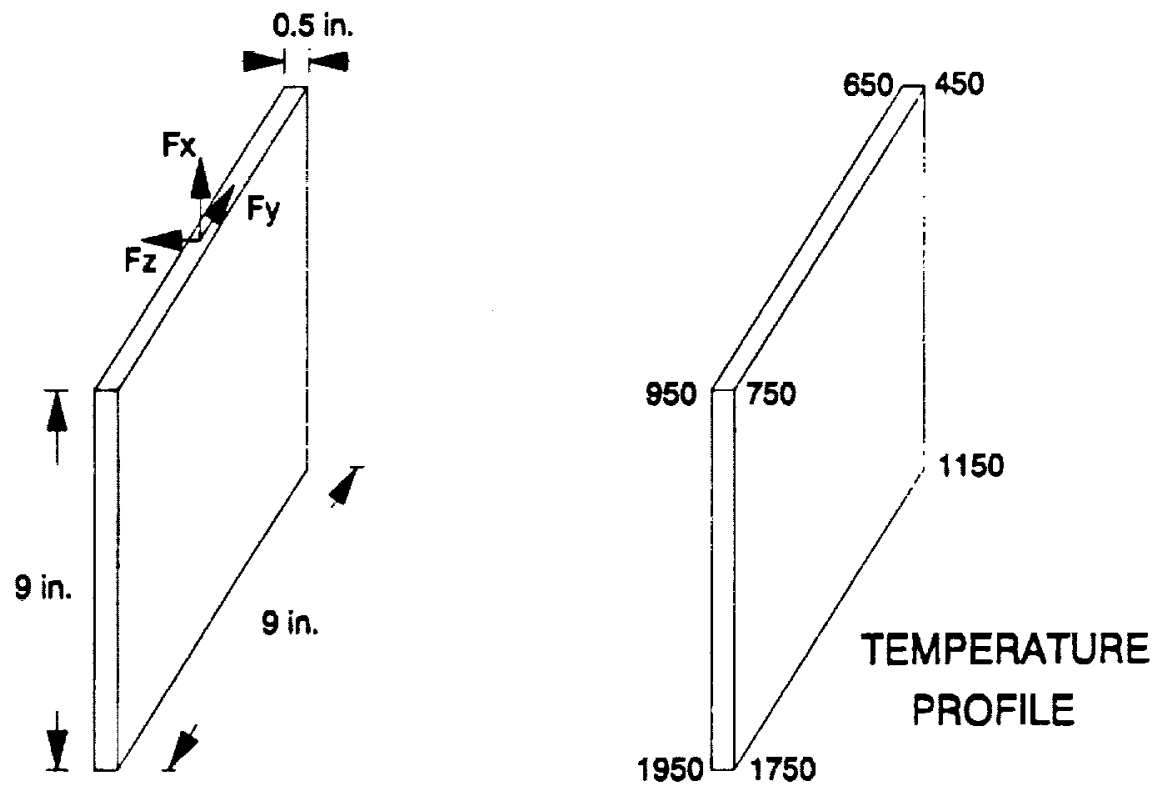

Fig. 6. The Geometry and Loading Conditions of a Cantilever plate (Example 3, temperatures in degree Fahrenheit) 
CUMULATIVE
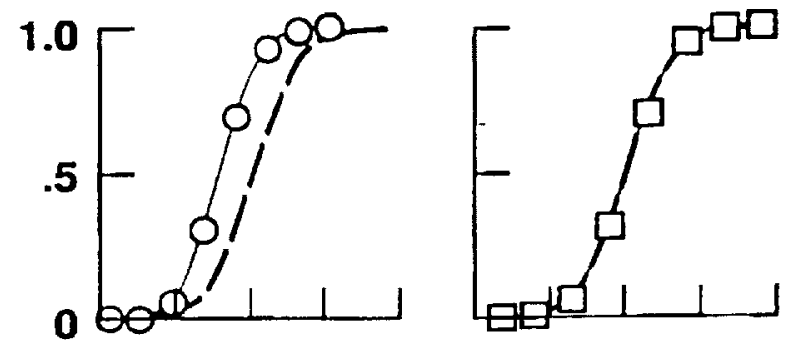

DISTRIBUTION

FUNCTION
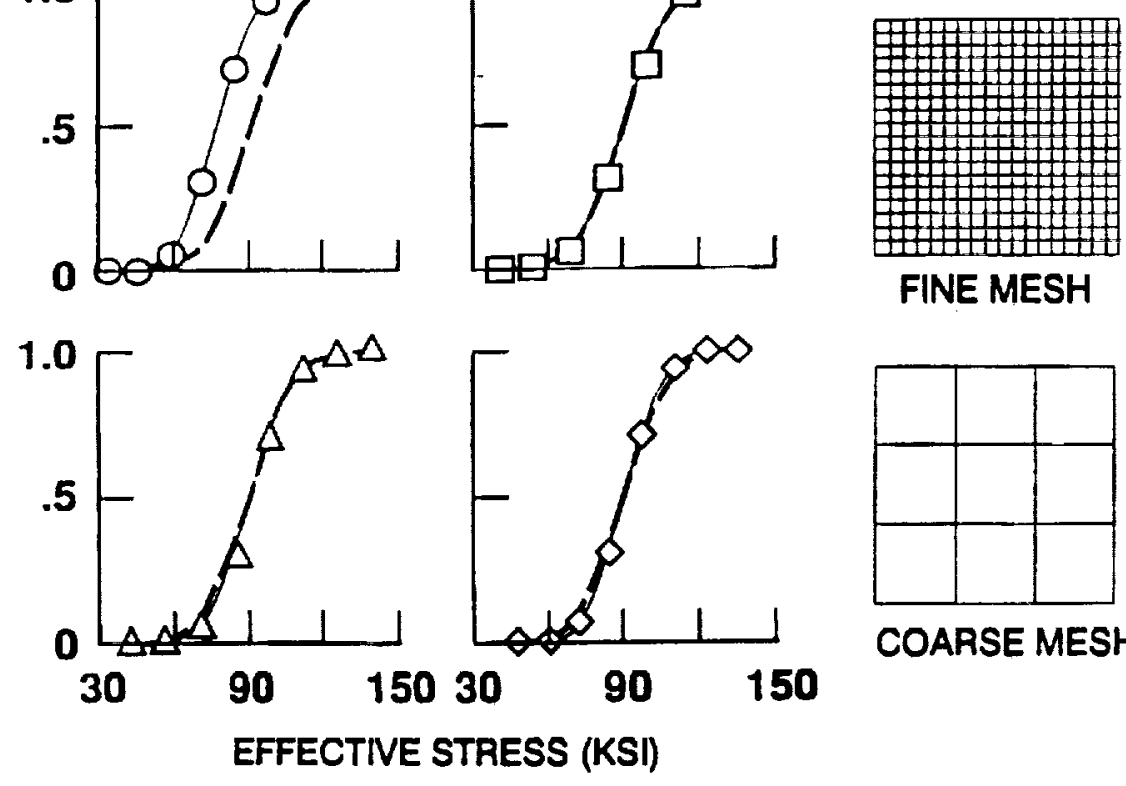

FINE MESH

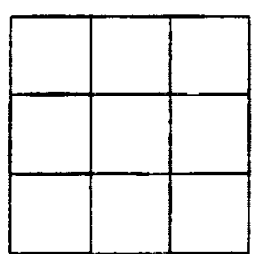

COARSE MESH

- - DINEMEOH

O COAREE MESH WTHOUT WAPANO

$\square$

CONGE MESH WTH MUTO MUPPINO

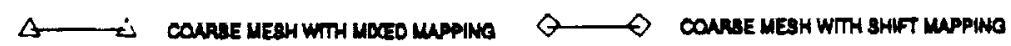

Fig. 7. Comparison Between the Cumulative Distribution Functions of the Effective Stress from Different Mapping Methods (Example 3)

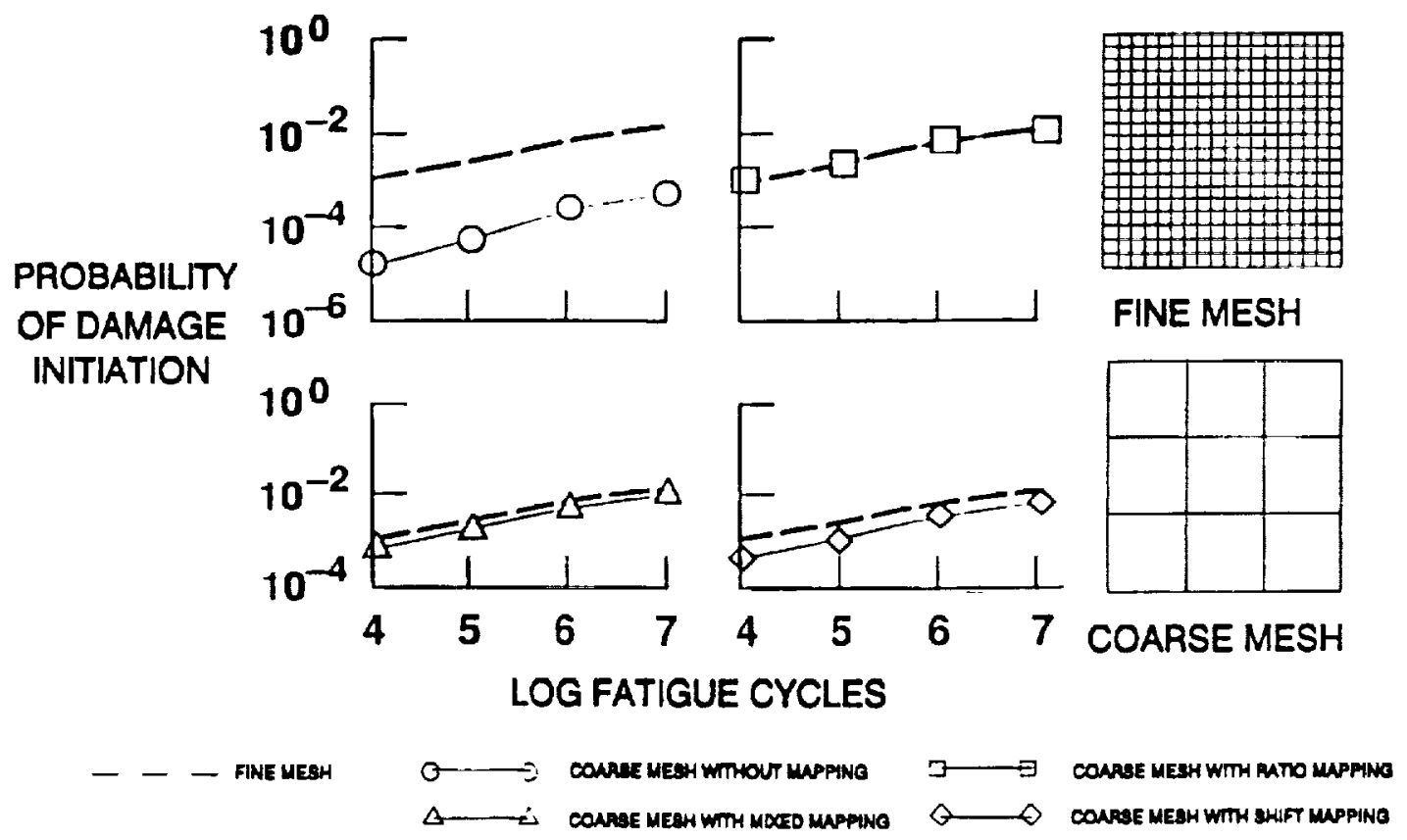

Fig. 8. Comparison Between the Probability of Damage Initiation from Different Mapping Methods (Example 3) 

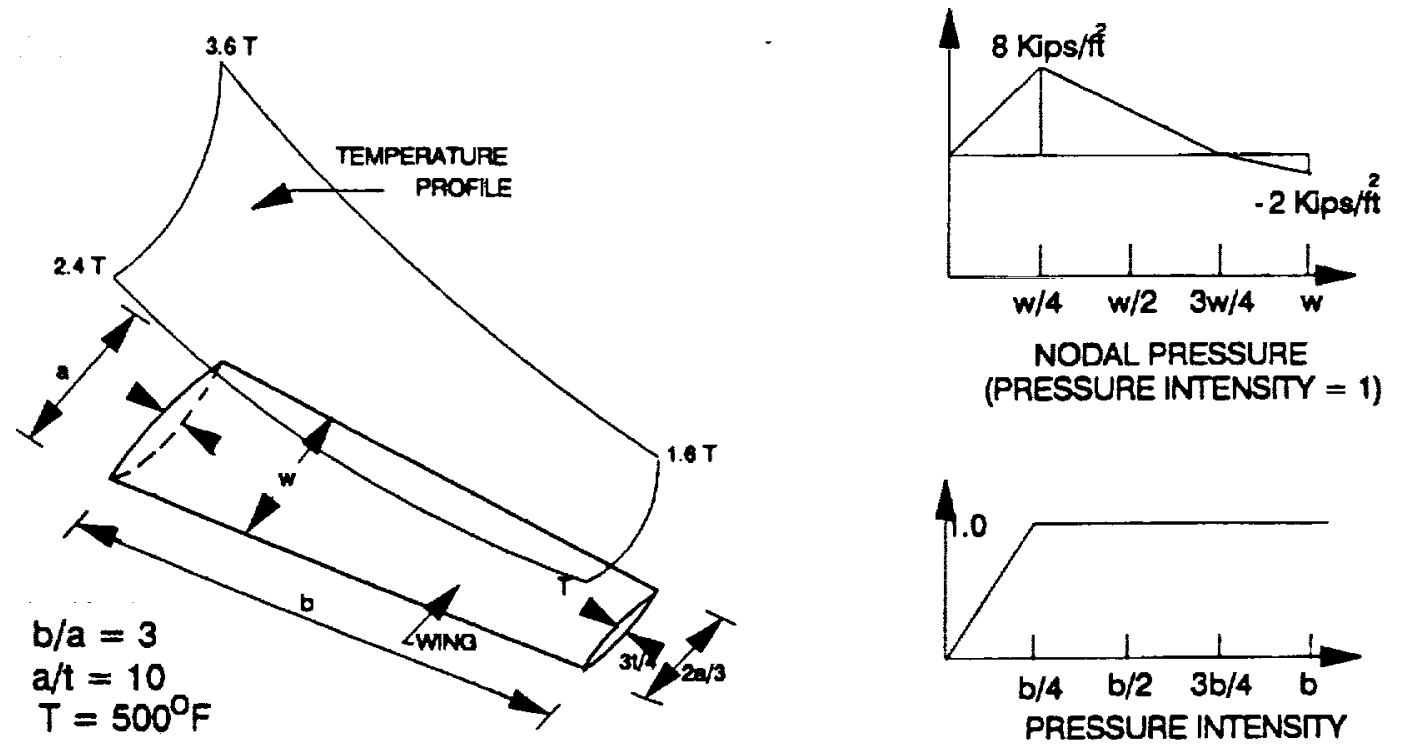

Fig. 9. Geometry and Loading Conditions for a Tapered Cantilever Plate (Example 4)

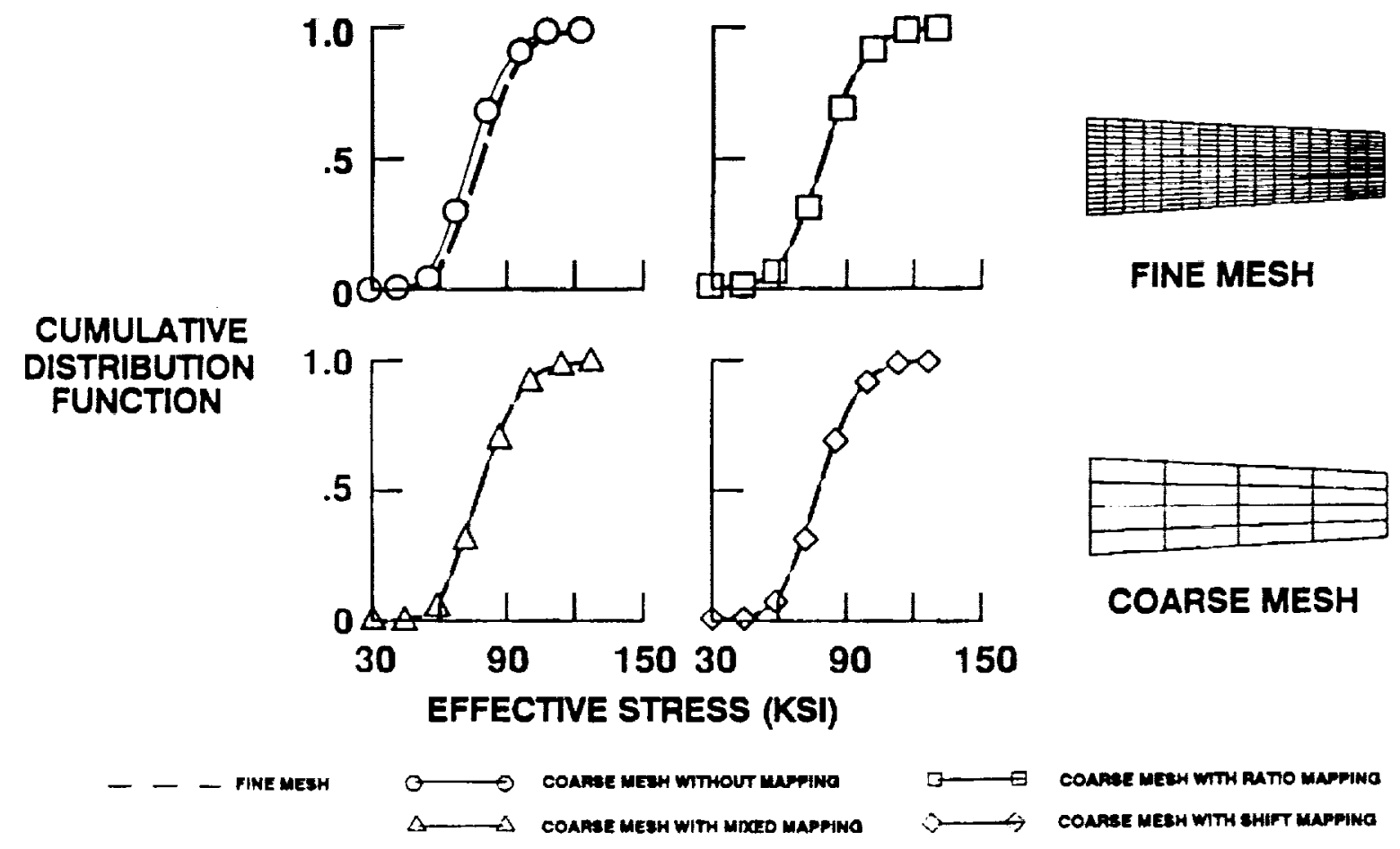

Fig. 10. Comparison Between the Cumulative Distribution Functions of the Effective Stress from Different Mapping Methods (Example 4) 
PROBABILTY OF DAMAGE INTIATION

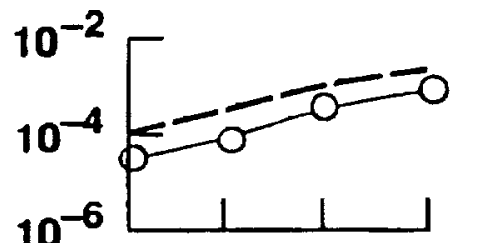

$10^{-2}$

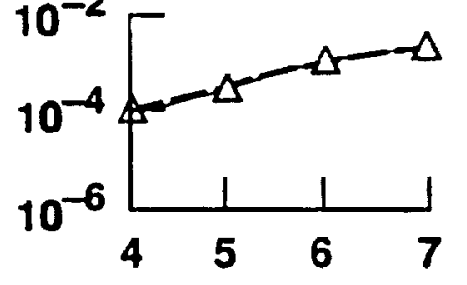

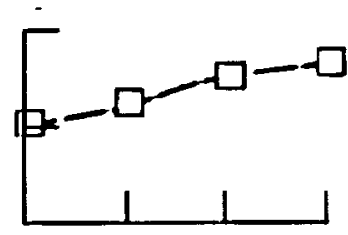

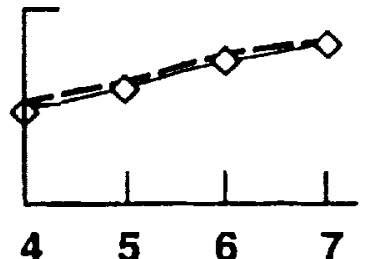

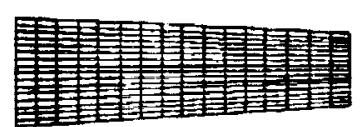

FINE MESH

\section{LOG FATIGUE CYCLES}

- - PINE MEsH

$$
\stackrel{0}{\triangle \longrightarrow}
$$

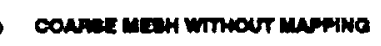

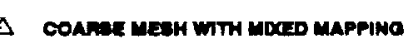

$\stackrel{\leftrightarrow}{\longrightarrow}$

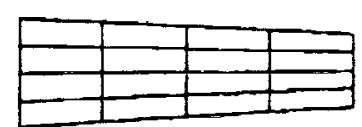

COARSE MESH

Fig. 11. Comparison Between the Probability of Damage Initiation from Different Mapping Methods (Example 4)

\begin{tabular}{|c|c|c|c|}
\hline $\begin{array}{c}\text { Number of } \\
\text { Nodes }\end{array}$ & $\begin{array}{c}\text { Deterministic } \\
\text { stress }\end{array}$ & $\begin{array}{c}\text { Error, } \\
\text { percent }\end{array}$ & $\begin{array}{c}\text { CPU time, } \\
\text { sec }\end{array}$ \\
\hline $16(4$ by 4$)$ & 75.4 & 16 & 0.9 \\
\hline $25(5$ by 5$)$ & 81.0 & 9 & 1.2 \\
\hline $36(6$ by 6$)$ & 83.9 & 6 & 1.7 \\
\hline $49(7$ by 7$)$ & 85.6 & 4 & 2.2 \\
\hline $64(8$ by 8$)$ & 86.7 & 3 & 2.9 \\
\hline $81(9$ by 9$)$ & 87.5 & 2 & 3.5 \\
\hline $100(10$ by 10$)$ & 88.1 & 1.5 & 4.5 \\
\hline $361(19$ by 19$)$ & 89.4 & 0 & 19.1 \\
\hline
\end{tabular}

Table 1. Comparison of the Error and CPU Time of the Deterministic Stress Using Different Finite Element Meshes (Example 3) 
Public reporting burden for this collection of information is estimated to average 1 hour per response. including the time for revlewing instructions, searching existing data sources, gathering and maintaining the data needed, and completing and reviewing the collection of information. Send comments regarding this burden estimate or any other aspect of this collection of information, including suggestions for reducing this burden, to Washington Headquarters Services. Directorate for information Operations and Reports. 1215 Jefferson Davis Highway, Sunte 1204, Arifington, VA 22202-4302, and 10 the Office of Management and Budget, Paperwork Feouction Project (0704-01a8), Washington, DC 20503.

\begin{tabular}{|l|c|c|}
\hline 1. AGENCY USE ONLY (Leave blank) & $\begin{array}{c}\text { 2. REPOAT DATE } \\
\text { October } 1992\end{array}$ & $\begin{array}{r}\text { 3. REPORT TYPE AND DATES COVEAED } \\
\text { Technical Memorandum }\end{array}$ \\
\hline
\end{tabular}

\section{TITLE AND SUBTITLE}

Mapping Methods for Computationally Efficient and Accurate Structural Reliability

\section{AUTHOR(S)}

Michael C. Shiao and Christos C. Chamis

$$
\text { WU }-553-13-00
$$

\section{PERFORMING ORgANIZATION NAME(S) AND ADDRESS(ES)}

8. PERFORMING ORganization REPORT NUMBER

National Acronautics and Space Administration

Lewis Research Center

Cleveland, Ohio 44135-3191

E-7359

9. SPONSORING/MONITORING AGENCY NAMES(S) AND ADDRESS(ES)

10. SPONSORING/MONITORING AGENCY REPORT NUMBER

National Aeronautics and Space Administration

Washington, D.C. 20546-0001

NASA TM- 105892

\section{SUPPLEMENTARY NOTES}

Prepared for the 33rd Structures, Structural Dynamics and Materials Conference, April 13-15, 1992, Dallas, Texas. Michacl C. Shiao, Sverdrup Technology, Inc., Lewis Research Center Group, 2001 Aerospace Parkway, Brook Park, Ohio 44142. Christos C. Chamis, Lewis Research Center. Responsible person, Michael C. Shiao, (216) 891-2986.

12.. DISTRIBUTION/AVAILABILITY STATEMENT

Unclassified - Unlimited

Subject Category 39

12b. DISTRIBUTION CODE

\section{ABSTRACT (Max/mum 200 words)}

Mapping methods are developed to improve the accuracy and efficiency of probabilistic structural analyses with coarse finite element meshes. The mapping methods consist of: (1) deterministic structural analyses with fine (convergent) finite element meshes, (2) probabilistic structural analyses with coarse finite element meshes, (3) the relationship between the probabilistic structural responses from the coarse and fine finite element meshes, and (4) a probabilistic mapping. The results show that the scatter in the probabilistic structural responses and structural reliability can be efficiently predicted using a coarse finite element model and proper mapping methods with good accuracy. Therefore, large structures can be efficiently analyzed probabilistically using finite element methods.

\section{SUBJECT TERMS}

Mapping; Reliability; Probability; Damage; Initiation; Structural; Structure; Fine; Coarse; Mesh; Finite element

17. SECURITY CLASSIFICATION OF REPORT Unclassified
10. SECURITY CLASSIFICATION OF THIS PAGE Unclassified
19. SECURITY CLASSIFICATHON OF ABSTRACT Unclassified
15. NUMBER OF PAGES 22

16. PRICE CODE $\mathrm{AO} 3$

20. LIMITATION OF ABSTRACT 